\title{
Effect of different doses of creatine supplementation on endogenous creatine synthesis
}

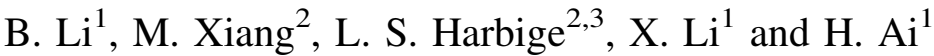 \\ ${ }^{1}$ Division of Nutrition and Biochemistry, Institute of Sports Medicine, The Third Hospital, Peking University, Beijing, \\ People's Republic of China, ${ }^{2}$ Centre for Biosciences Research, School of Science, University of Greenwich, Kent ME4 $4 T B$, \\ $U K$ and ${ }^{3}$ Medway School of Pharmacy, University of Kent and University of Greenwich, Kent ME4 4TB, UK
}

Creatine (Cre) has been widely used as a nutritional ergogenic aid among athletes since the middle of the 1990s ${ }^{(1)}$ but has not been categorised as a stimulant. Athletes often take a greater dose of Cre for a longer period in order to obtain a rapid, high and longer ergogenic effect ${ }^{(2)}$. L-Arginine:glycine amidinotransferase (L-AGAT) in the kidney is regarded as a key enzyme in endogenous Cre synthesis in mammals, and guanidinoacetic acid (GAA) is the precursor in this synthesis ${ }^{(3)}$. The present study has investigated the effect of supplementation with $0,0.75,1.5,3.0$ and $6.0 \mathrm{~g}$ Cre/kg body weight per d for 4 weeks on L-AGAT activity and GAA concentration in the kidneys and total Cre in the gastrocnemius muscle in adult male Sprague-Dawley rats. L-AGAT activity and GAA concentration in the kidneys decreased $(\%)$ by $19.5,42.6,61.9$ and 66.5 , and 5.9, 13.7, 23.8 and 24.5 respectively in the groups supplemented with $0.75,1.5$, 3.0 and $6.0 \mathrm{~g} \mathrm{Cre} / \mathrm{kg}$ perd for 4 weeks. The total Cre in the gastrocnemius in the supplemented groups increased $(\%)$ by $7.1,8.5,12.6$ and 13.7 respectively. The weight of the gastrocnemius in the groups receiving $0.75,1.5$ and $3.0 \mathrm{~g} \mathrm{Cre} / \mathrm{kg}$ per $\mathrm{d}$ also increased significantly (see Table). No significant differences were found in the body weights among the groups supplemented with $0,0.75,1.5$ and $3.0 \mathrm{~g}$ Cre/kg per $\mathrm{d}$ for 4 weeks (Table). However, the body weight of the group receiving $6.0 \mathrm{~g} \mathrm{Cre} / \mathrm{kg}$ per d was significantly less than that of the control group (Table). After $3 \mathrm{~d}$ of supplementation diarrhoea was observed in all the rats in the group receiving $6.0 \mathrm{~g}$ Cre/kg per $\mathrm{d}$, and persisted for the remainder of the supplementation period, which probably explains the loss in body weight. No significant differences were found in the serum Cre kinase activity and serum creatinine among the five groups. L-AGAT activity was positively correlated with the concentration of GAA ( $n$ 50, $r 0.80, P<0.001)$. The Cre intake was, however, negatively correlated with the L-AGAT activity $(n$ 50, $r 0.87, P<0.001)$ and the concentration of GAA $(n 50, r 0.75, P<0.001)$.

\begin{tabular}{|c|c|c|c|c|c|}
\hline \multirow[b]{2}{*}{ Cre $(g / k g$ per $d)$} & \multirow[b]{2}{*}{$n$} & \multicolumn{2}{|c|}{ Body weight $(\mathrm{g})$} & \multicolumn{2}{|c|}{ Gastrocnemius weight (g) } \\
\hline & & Mean & SD & Mean & SD \\
\hline 0 & 10 & 365.2 & 36.0 & 2.02 & 0.17 \\
\hline 0.75 & 10 & 379.4 & 23.7 & $2.33 * *$ & 0.24 \\
\hline 1.5 & 10 & 374.8 & 19.6 & $2.19^{*}$ & 0.10 \\
\hline 3.0 & 10 & 371.8 & 21.8 & $2.27 * *$ & 0.20 \\
\hline 6.0 & 10 & $340.6 * *$ & 31.0 & 2.05 & 0.19 \\
\hline
\end{tabular}

Mean values were significantly different from that for the control group (without Cre supplementation): $* P<0.05$, $* * P<0.01$.

These results indicate that in rats L-AGAT activity and GAA concentration could be rapidly reduced by supplementation with $0.75-$ $3.0 \mathrm{~g} \mathrm{Cre} / \mathrm{kg}$ per d, which is equivalent to $10-40 \mathrm{~g}$ Cre/d $\left(67 \mathrm{~kg}\right.$ body weight) in human subjects ${ }^{(4)}$, suggesting that high-dose Cre supplementation may result in depression of endogenous Cre metabolism and may have potential adverse effects on the body. Little is known about the effect of high-dose Cre supplementation on the immune system, the results of a follow-up study of immune function will be of interest.

1. Bemben MG \& Lamont HS (2005) Sports Med 35, 107-125.

2. Brudnak MA (2004) Toxicol Lett 150, 123-130.

3. Wyss M \& Kaddurah-Daouk R (2000) Physiol Rev 80, 1107-1213

4. Calabrese EJ (1991) Principles of Animal Extrapolation, pp. 499-527. Chelsea, MI: Lewis Publishers Inc. 